\title{
Thought Experiment to Examine Benchmark Performance for Fusion Nuclear Data
}

\author{
Isao Murata ${ }^{1, a}$, Masayuki Ohta ${ }^{2}$, Sachie Kusaka ${ }^{1}$, Fuminobu Sato $^{1}$, Hiroyuki Miyamaru ${ }^{3}$ \\ ${ }^{1}$ Division of Electrical, Electronic and Information Engineering, Osaka University, Yamada-oka 2-1, Suita, Osaka, 565-0871 Japan \\ ${ }^{2}$ Center for Atomic and Molecular Technology, Osaka University, Yamada-oka 2-1, Suita, Osaka, 565-0871 Japan \\ ${ }^{3}$ Organization for Industry, University and Government Cooperation, Osaka Prefecture University, Sakai, Osaka 599-8570, Japan
}

\begin{abstract}
There are many benchmark experiments carried out so far with DT neutrons especially aiming at fusion reactor development. These integral experiments seemed vaguely to validate the nuclear data below 14 $\mathrm{MeV}$. However, no precise studies exist now. The author's group thus started to examine how well benchmark experiments with DT neutrons can play a benchmarking role for energies below $14 \mathrm{MeV}$. Recently, as a next phase, to generalize the above discussion, the energy range was expanded to the entire region. In this study, thought experiments with finer energy bins have thus been conducted to discuss how to generally estimate performance of benchmark experiments. As a result of thought experiments with a point detector, the sensitivity for a discrepancy appearing in the benchmark analysis is "equally" due not only to contribution directly conveyed to the deterctor, but also due to indirect contribution of neutrons (named (A)) making neutrons conveying the contribution, indirect controbution of neutrons (B) making the neutrons (A) and so on. From this concept, it would become clear from a sensitivity analysis in advance how well and which energy nuclear data could be benchmarked with a benchmark experiment.
\end{abstract}

\section{Introduction}

There are a lot of nuclear data measurements, named differential experiments, carried out so far with DT neutron sources. Also there exist many benchmark experiments, i.e., integral experiments, to verify the nuclear data validity. They are of course aiming at fusion reactor development finally. Differential experiments can check the accuracy of evaluated nuclear data at 14 $\mathrm{MeV}$ directly. Integral experiments are vaguely expected to validate the nuclear data also below $14 \mathrm{MeV}$ as well as at $14 \mathrm{MeV}$. However, no precise studies concerning this matter were performed systematically so far. On the other hand, as for numerical calculations with transport codes, it is quite common to analyze benchmark experiments using Monte Carlo codes. It would become very useful if benchmark performance for nuclear data could directly be analyzed beforehand from the Monte Carlo calculations. In the author's group, various fusion neutronics experiments have been carried out so far with a $14 \mathrm{MeV}$ neutron source, OKAVIAN. We thus started to examine how well integral benchmark experiments with DT neutrons can play a benchmarking role for energies below $14 \mathrm{MeV}$ using a general purpose Monte Carlo code MCNP. ${ }^{1)}$

We calculated an energy spectrum of neutrons making neutrons or gamma-rays detected by a detector in a leakage neutron or gamma-ray spectrum measurement. This spectrum was named "neutron spectrum before last collision", $\varphi_{\text {last, }}$ in this study. Calculation of $\varphi_{\text {last }}$ was done with a point detector as in the following procedure. For each detected contribution, there exists a neutron or gamma-ray, named (A), conveying the contribution to the detector. The information, i.e., the contribution and energy of (A) is summed up to make a measured spectrum in the calculation. Now, we think of another neutron (B), making the neutron or gamma-ray, $(\mathrm{A})$, conveying the contribution. The spectrum of $\varphi_{\text {last }}$ can be evaluated by contribution conveyed by particle (A) with the energy of particle (B) instead of (A).

As a result of the series study, ${ }^{2,3)}$, especially for DT neutron benchmark experiments, it was found that for secondary gamma-ray spectrum measurements nuclear data at around $14 \mathrm{MeV}$ were dominantly benchmarked. In return, for neutron spectrum measurements those below $14 \mathrm{MeV}$ were also benchmarked fairly well in addition to $14 \mathrm{MeV}$, because neutrons could rapidly be moderated in a sample material. In conclusion, to make benchmark experiments efficient, a spectrum shifter made with beryllium should be used to make the incident neutron spectrum softer especially for gamma-ray spectrum measurements. For neutron spectrum measurements, it was pointed out that reduction of experimental time could be expected.

a Corresponding author: murata@see.eng.osaka-u.ac.jp 
In benchmark analyses carried out so $\operatorname{far}^{2,3}$, we set only one energy boundary at $10 \mathrm{MeV}$ to examine $\varphi_{\text {last }}$, meaning the number of energy groups is just two (14 $\mathrm{MeV}$ and other than $14 \mathrm{MeV}$ ), because the incident particle is a mono-energetic $14 \mathrm{MeV}$ neutron and we wanted to know simply if the measured neutron spectrum below $14 \mathrm{MeV}$ could validate the nuclear data not only for $14 \mathrm{MeV}$ but also below $14 \mathrm{MeV}$. This concept worked well for performance analysis of the benchmark with DT neutrons. But in general cases it seemed to be insufficient to employ one boundary. The key point is how we should make a discussion in the general cases in the same manner as the case of two energy groups. As shown in Fig. 1 which is the case of a leakage neutron spectrum by DT neutrons, it is found that neutrons (B) are already staying in a bin close to the energy bin of neutrons (A). In this sample size (about several tens $\mathrm{cm}$ thick), it can be found that the neutron spectrum mostly reaches its equilibrium one. For gammray spectrum case in Fig. 2, in contrary, gamma-rays produced by $14 \mathrm{MeV}$ neutrons are found to be dominant. It means especially for neutrons that finer energy meshes are essential and indispensable in general cases. Consequently, two problems were pointed out to discuss the benchmark performance in Ref. 3).

Here, one of the two is introduced, because it is directly related to the present objectives. In our previous studies the problem did not explicitly appear, because the energy boundary is only at $10 \mathrm{MeV}$, meaning that only $14 \mathrm{MeV}$ neutrons and neutrons below $14 \mathrm{MeV}$ exist. However, if using finer energy bins, there must exist various energetic neutrons, i.e., neutrons (A) conveying contribution to a certain energy mesh of the spectrum, neutrons (B) making neutrons (A), neutrons (C) making neutrons (B) and so on. The key issue is that one has to think of not only neutrons (A) but also other neutrons created during the whole particle history from the source.

In the present study, we carry out a thought experiment to precisely examine which neutron, i.e., nuclear data for the neutron energy, created during a transport history is benchmarked by the measured detector contribution. The change appearing in the measured spectrum is examined, if the cross section value were artificially changed to be a little over- or underestimated. From the result, the benchmark performance sensitivity due to discrepancy between measurement and calculation is discussed. Investigating the whole thought experiment, we discuss how well neutrons created during the transport are benchmarked. And finally we examine that we reach the conclusion that there would be no need to calculate adjoint function for benchmark performance (sensitivity) analysis.

\section{Thought Experiment}

As a thought experiment, we think of a DT neutron incidence to a sample as shown in Fig. 3. The sample is so thin that it can be assumed that scattering occurs just twice at maximum. Also, assuming that emitted secondary neutrons via first and second scattering can be measured separately. It means that the spectrum from the first scattering corresponds to double differential cross section (DDX) and the second one is a distortion term by scattering. The mixture of the two is a neutron spectrum to be measured.

In the separate measuremtn, we think of how effectively each spectrum plays a benchmarking role. Naturally, the neutron spectrum made directly from the first scattering plays a benchmarking role for the incident $14 \mathrm{MeV}$ neutrons $\left(\mathrm{n}_{0}\right)$. In this case the contribution is conveyed by neutrons $\left(\mathrm{n}_{1}\right)$ to the detector. The second spectrum is made up of contributions created by neutrons $\left(n_{1}\right)$ emitted via nuclear reactions of the incident neutrons $\left(\mathrm{n}_{0}\right)$. The contributions are delivered by neutrons $\left(\mathrm{n}_{2}\right)$. Therefore, the spectrum can play a benchmarking role for $\mathrm{n}_{1}$. The point is 'whether the second one could have a benchmarking role also against the incident neutrons $\left(\mathrm{n}_{0}\right)$ '. Seemingly, it is quite likely, because neutrons $\left(\mathrm{n}_{1}\right)$ producing the second spectrum are originally created by neutrons $\left(\mathrm{n}_{0}\right)$. Now, the problem is, if so, 'whether the benchmark performance degree for both of $\mathrm{n}_{0}$ and $\mathrm{n}_{1}$ is the same'.

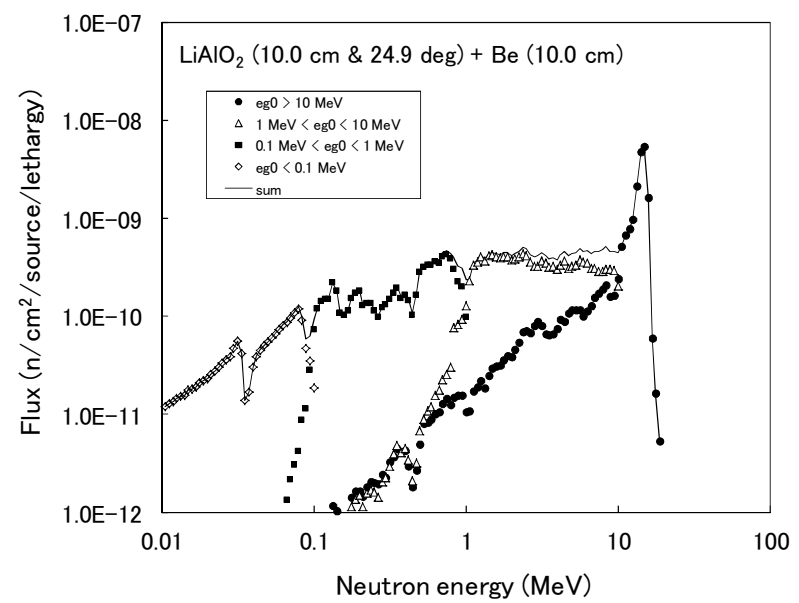

Fig. 1. Calculated leakage neutron spectrum for $\mathrm{Li}_{2} \mathrm{TiO}_{3}$ by DT neutrons. 'eg0' is the energy of neutron spectrum before last collision, $\varphi_{\text {last, }}$ and 'sum' is the calculated neutron spectrum. $\boldsymbol{Q}, \triangle, \boldsymbol{\square}$ and $\diamond$ indicate to partial spectra made by each $\varphi_{\text {last. }}$

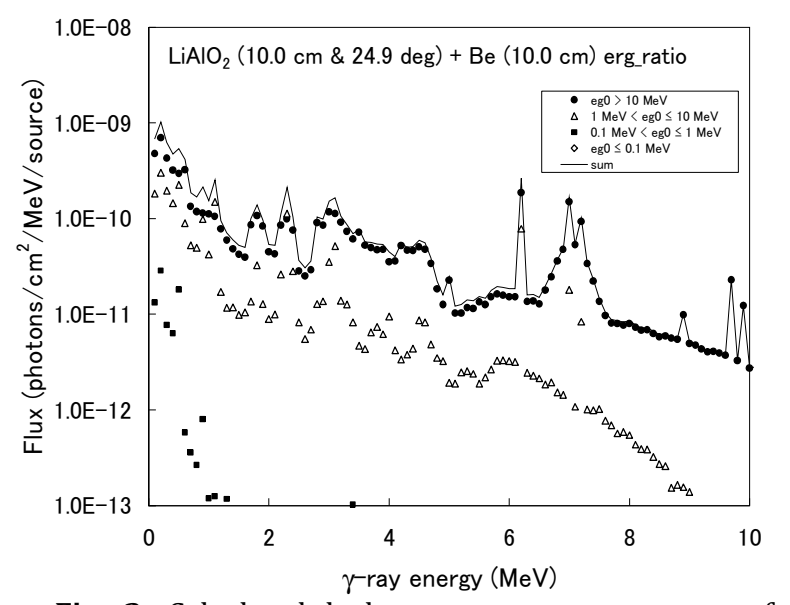

Fig. 2. Calculated leakage gamma-ray spectrum for $\mathrm{Li}_{2} \mathrm{TiO}_{3}$ by DT neutrons. Other condition is the same as Fig. 1. 
Table 1 Error sensitivity analysis result in a simple benchmark model.

\begin{tabular}{|c|c|c|c|c|c|}
\hline \multirow{2}{*}{} & \multicolumn{2}{|c|}{ Cross section assumption } & \multicolumn{2}{c|}{ Comparison of experiment and calculation } \\
\cline { 2 - 5 } & $14 \mathrm{MeV}$ & Below $14 \mathrm{MeV}$ & $\mathrm{n}_{1}$ spectrum & $\mathrm{n}_{2}$ spectrum & Total \\
\hline$(1)$ & $-10 \%{ }^{*}$ & Correct & $-10 \%{ }^{* 2}$ & $-10 \%$ & $-10 \%$ \\
\hline$(2)$ & Correct & $-10 \%$ & Correct & $-10 \%$ & $\begin{array}{c}\text { Underestimated } \\
\text { a little }\end{array}$ \\
\hline$(3)$ & $-5 \%$ & $-5 \%$ & $-5 \%$ & $-5 \sim-10 \%$ & $-5 \sim-10 \%{ }^{* 4}$ \\
\hline
\end{tabular}

*1 Cross section is assumed to be underestimated. *2 Underestimation of calculation

*3 Because $\mathrm{n}_{1}$ spectrum is dominant. *4 The value is expected to be close to $-5 \%$.

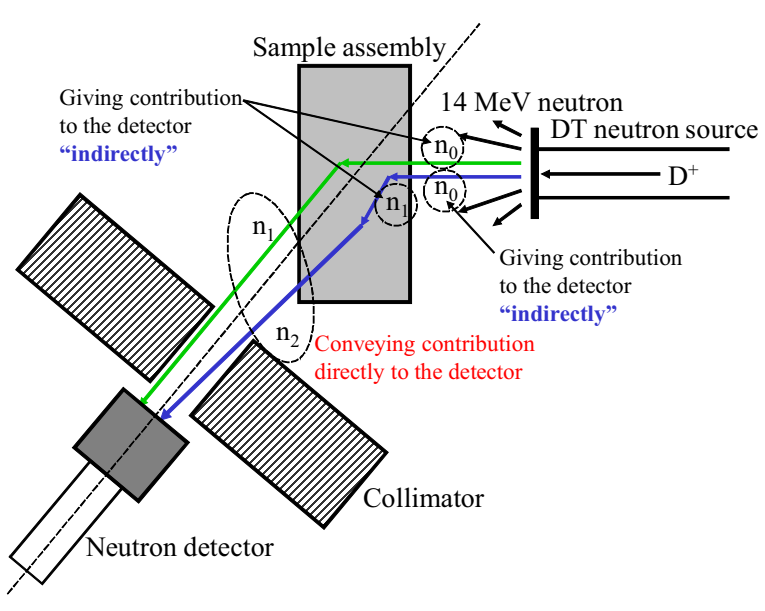

Fig. 3 Schematic arrangement of neutron benchmark experiment for the thought experiment.

\subsection{Simple benchmark sensitivity}

Now thinking of a similar benchmark experiment to Fig. 2 with a relatively thin slab sample, the benchmark sensitivity is examined with a simple condition as follows: Assuming cross sections except $14 \mathrm{MeV}$ are correct, and that of $14 \mathrm{MeV}$ is underestimated by $10 \%$ (By this, the produced DDX by $14 \mathrm{MeV}$ neutron shows a little smaller spectrum by $10 \%$.) In this case, if comparing the measured spectrum with the calculation, the calculated $n_{1}$ spectrum shows $10 \%$ smaller in the whole energy region. This means that the number of neutrons making the second spectrum is decreased by 10 $\%$. Thus, the spectrum of $\mathrm{n}_{2}$ neutrons produced by $\mathrm{n}_{1}$ shows $10 \%$ smaller also. As a total of $n_{1}$ and $n_{2}$, the estimated measured spectrum shows $10 \%$ smaller. The result is summarized in Table 1 . The above result indicates that both spectra due to the first and second scatterings play equally the benchmarking role at the energy of $14 \mathrm{MeV}$.

Inversely, assuming the cross section of $14 \mathrm{MeV}$ is correct and others below $14 \mathrm{MeV}$ are smaller by $10 \%$, a similar simple thought experiment is done as follows: The $\mathrm{n}_{1}$ spectrum calculated is clearly correct (showing the same as the measurement). As for the $\mathrm{n}_{2}$ spectrum, the absolute value becomes smaller by $10 \%$ because of $10 \%$ smaller cross sections for neutrons $\mathrm{n}_{1}$, although the intensity of neutrons $\mathrm{n}_{1}$ (produced as a result of the first scattering by $\mathrm{n}_{0}$ ) making the $\mathrm{n}_{2}$ spectrum is correct. The summed-up spectrum to be measured shows a smaller value by less than $10 \%$, probably the absolute value is smaller, because in reality the spectrum of $n_{1}$ is dominant.

Then, in case of the cross section being $5 \%$ smaller in both energy ranges, meaning $5 \%$ smaller in the whole energy range, the $\mathrm{n}_{1}$ spectrum shows $5 \%$ smaller. Because the spectrum of neutrons making the $n_{2}$ spectrum is also $5 \%$ smaller, the $\mathrm{n}_{2}$ spectrum has to be smaller by $5 \%$. The total result shows a decrease of between 5 and $10 \%$, probably close to $-5 \%$.

In summary, when the calculated spectrum shows a $10 \%$ smaller value in energies below the elastic peak of $14 \mathrm{MeV}$ compared to an experiment, the benchmark activity is to find out the reason why the discrepancy happens. From the above sensitivity analysis, it would be the most likely that the cross section of $14 \mathrm{MeV}$ would be wrong (smaller), then second-likely that both would be underestimated, and the probability is the smallest that the cross sections only below $14 \mathrm{MeV}$ are incorrect.

The important conclusion is that this kind of evaluation is of course not possible if only neutron spectrum before last collision is taken into consideration.

\subsection{Precise benchmark sensitivity}

Based on the results obtained above, a more precise benchmark sensitivity is next examined with a practical model, that is, a leakage spectrum measurement with a slab assembly by $14 \mathrm{MeV}$ neutron incidence. Here, we focus on an energy bin of 5 to $6 \mathrm{MeV}$, for example, in the measured spectrum. Similar to the previous section, the number of scatterings is assumed to be up to twice. In this case, some measured neutrons may be produced by $14 \mathrm{MeV}$ neutron's nuclear reactions, and some others may be via nuclear reactions by neutrons below $14 \mathrm{MeV}$, which are created by the $14 \mathrm{MeV}$ neutron's nuclear reactions. The former measured neutrons are named $n_{1}$, and the latter is $\mathrm{n}_{2}$, in the same manner as the previous section. Now, we employ the following assumption additionally that the ratio of the detector contribution in the energy bin conveyed by $\mathrm{n}_{1}$ is $1-a(0<a<1)$, and that by $\mathrm{n}_{2}$ is $a$ as shown in Fig. 4. So, if the C/E is 0.9 and the error contribution is simply proportional to contribution of the whole spectrum, it could be expected 
Table 2 Error sensitivity analysis result in a precise benchmark model.

\begin{tabular}{|c|c|c|c|c|c|c|}
\hline Measured & $\mathrm{n}_{1}$ spectrum & $\mathrm{n}_{2}$ spectrum & $\begin{array}{c}\text { Error sensitivity } \\
\text { to the energy bin }\end{array}$ & Total & Ratio & Expectation \\
\hline \multicolumn{7}{|c|}{$10 \%$ underestimated nuclear data at $14 \mathrm{MeV}$ and correct for others to examine $\mathrm{n}_{0}$ sensitivity } \\
\hline $\mathrm{n}_{1}$ & $-10 \%$ & - & $-10(1-a) \%$ & \multirow{2}{*}{$-10 \%{ }^{*}$} & 1 & $1-a$ \\
\hline $\mathrm{n}_{2}$ & $-10 \%{ }^{* 1}$ & $-10 \%$ & $-10 a \%$ & & \\
\hline \multicolumn{7}{|c|}{ Correct nuclear data for $14 \mathrm{MeV}$ and $10 \%$ underestimated for others to examine $\mathrm{n}_{1}$ sensitivity } \\
\hline $\mathrm{n}_{1}$
\end{tabular}

*1 As a source to make $\mathrm{n}_{2} . \quad * 2 \mathrm{n}_{0}$ sensitivity $* 3 \mathrm{n}_{1}$ sensitivity

that the origin of the error, $10 \%$, could be divided into $10(1-a) \%$ for $14 \mathrm{MeV}$ neutrons and $-10 a \%$ for $\mathrm{n}_{1}$, because $n_{1}$ is produced by a $14 \mathrm{MeV}$ neutron and $n_{2}$ is produced by $n_{1}$. However, as detailed in the following, it will be made clear that this is not the case.

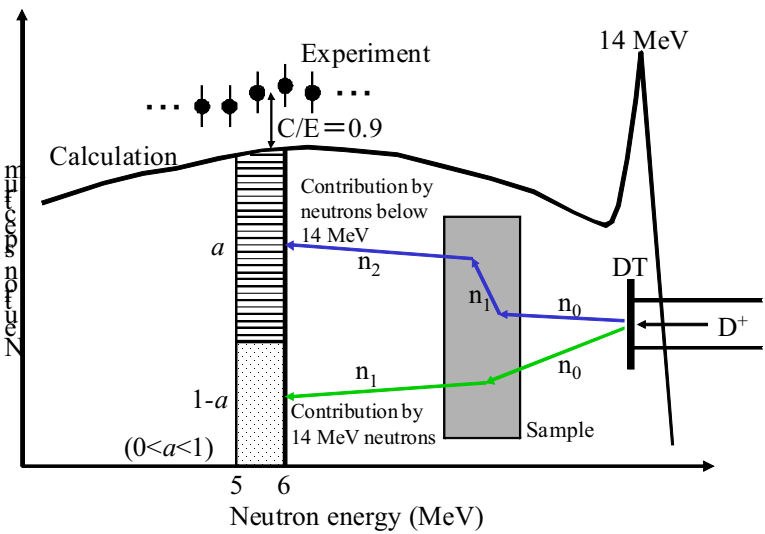

Fig. 4 Detector contribution ratio $a$ in neutron spectrum.

In the condition above, from the result in Sec. 2.1, it can be expected that the probability is larger that the cross section of $14 \mathrm{MeV}$ is incorrect. In other words, the crucial point is that $\mathrm{n}_{1}$ is the source term to make $\mathrm{n}_{2}$. Again we think of the condition, $\mathrm{C} / \mathrm{E}=0.9$.

At first it is assumed that the cross section at $14 \mathrm{MeV}$ is underestimated by $10 \%$ and others are correct. In this case, as shown in Table 2 , the calculated $n_{1}$ spectrum shows an underestimated result by $-10 \%$, and the error sensitivity of bin $5 \sim 6 \mathrm{MeV}$ due to $\mathrm{n}_{1}$ is estimated to be $10(1-a) \%$. Though cross sections of scattering induced by $\mathrm{n}_{1}$ are correct, the spectrum of $\mathrm{n}_{1}$ as a source term for the following nuclear reaction is smaller by $-10 \%$. The calculated spectrum of $\mathrm{n}_{2}$ thus becomes $-10 \%$, because other cross sections than $14 \mathrm{MeV}$ are assumed to be correct. Consequently, The error sensitivity of bin $5 \sim 6$ $\mathrm{MeV}$ due to $\mathrm{n}_{2}$ is $-10 a \%$. As a result, the total sensitivity of the bin is calculated by summing up $\mathrm{n}_{1}$ and $\mathrm{n}_{2}$ error sensitivities, i.e., $-10 \%$.

Second, it is assumed that the cross section at 14 $\mathrm{MeV}$ is correct and others are underestimated by $10 \%$. In this case, the calculated spectrum of $\mathrm{n}_{1}$ is correct, and the error sensitivity of bin $5 \sim 6 \mathrm{MeV}$ due to $\mathrm{n}_{1}$ is estimated to be zero. The spectrum of $n_{1}$ as a source term for the following nuclear reaction is also correct. However, the spectrum of $n_{2}$ possesses $-10 \%$ error, because other cross sections than $14 \mathrm{MeV}$ are assumed to be underestimated by $10 \%$. The error sensitivity of bin $5 \sim 6 \mathrm{MeV}$ due to $\mathrm{n}_{2}$ is thus $-10 a \%$ and the total sensitivity of the bin is thus $-10 a \%$.

As a sensitivity ratio, $14 \mathrm{MeV}:$ Others $=1: a$, meaning the probability is higher that the cross section at $14 \mathrm{MeV}$ is not correct, as expected also in Sec. 2.1. However, as described earlier it was expected the ratio is $1-a: a$. Both conclusions do not agree with each other. In the next section, the reason why the disagreement appears will be made clear.

\subsection{Benchmark sensitivity contribution of each scattering neutron}

In the beginning of Sec. 2.2, out of the contribution in the bin 5 6 MeV, it was seemingly expected that the ratio of $1-a$ came from $\mathrm{n}_{1}$ and ratio, $a$, from $\mathrm{n}_{2}$. However, this was available in case that we focused just on neutrons before last collision. In the conclusion of Sec. 2.2, considering another nuclear reaction forming spectrum $\mathrm{n}_{2}$, the error sensitivity became

$$
14 \mathrm{MeV}: \text { Others }=1: a
$$

In fact, this sensitivity is deeply related to the place where detector contribution comes from. Practically, $\mathrm{n}_{2}$ surely conveys contribution $a$ in the energy bin $5 \sim 6$ as shown in Fig. 4. However, $\mathrm{n}_{2}$ is created by $\mathrm{n}_{1}$ via nuclear reaction. It means that it can be said that $\mathrm{n}_{1}$ would convey the contribution indirectly to the detector. More precisely, it can be concluded from a physical consideration with 'importance' proposed by Lewins ${ }^{4)}$ with the result in Table 2 that "Error sensitivity for a certain neutron is proportional to the total detector contribution neutrons give directly and indirectly." Concretely, the detector contribution $14 \mathrm{MeV}$ neutrons, $\mathrm{n}_{0}$, give includes partly contribution, $1-a$, through neutron $\mathrm{n}_{1}$ as shown in Table 3. This is a direct contribution made by a $14 \mathrm{MeV}$ neutron. And there is another contribution of $\mathrm{n}_{1}, a$, conveyed by $\mathrm{n}_{2} . \mathrm{n}_{2}$ is made by $n_{1}$ 's nuclear reaction. This is also a direct contribution of $n_{1}$ conveyed by $n_{2}$. Since $n_{1}$ is created by $n_{0}$, it can be thought that $\mathrm{n}_{0}$ has an indirect contribution $a$. This is the same concept as 'importance' proposed by Lewins. As a 
result, $\mathrm{n}_{0}$ has direct contribution, $1-a$, and indirect contribution, $a$, leading to the conclusion that $\mathrm{n}_{0}$ has the total contribution,

$$
(1-a)+a=1
$$

On the other hand, the contribution of other neutrons, $\mathrm{n}_{1}$, is just $a$, directly made by themselves. Consequently, the detector contribution ratio can be estimated to be

$$
14 \mathrm{MeV}: \text { Others }=1: a
$$

as summarized in Table 3. This result is surprisingly the same as the error sensitivity ratio discussed in Sec. 2.2 and shown in Table 2.

Now, the problem is why the above expectation holds. The error sensitivity of $14 \mathrm{MeV}$ neutrons in the case of assuming cross sections except $14 \mathrm{MeV}$ are correct and that of $14 \mathrm{MeV}$ is underestimated by $10 \%$, described in the previous section, shows

$$
-10(1-a) \%+(-10 a \%)=-10 \%
$$

It seems that a sensitivity of neutrons other than 14 $\mathrm{MeV}$, that is, $-10 a \%$ is added. This is just caused by the fact that $14 \mathrm{MeV}$ neutrons produce $\mathrm{n}_{1}$. More practically, underestimation of cross section at $14 \mathrm{MeV}$ propagates in the lower energy neutrons, $\mathrm{n}_{1}$, created by $\mathrm{n}_{0}$. This is the indirect contribution. If removing an initially given error of $-10 \%$, the total ratio unity in Table 2 is exactly the same as the contribution summation calculation just above. On the other hand, the error sensitivity of other neutrons $\mathrm{n}_{1}$ in Sec. 2.2 is $-10 a \%$. This just means if removing $-10 \%$, the term $a$ is left, which is similarly the same as the contribution in Table 3.

Table 3 Direct/indirect contribution ratio to the detector.

\begin{tabular}{c|ccc}
\hline Contribution & Direct & Indirect & Total \\
\hline $14 \mathrm{MeV}\left(\mathrm{n}_{0}\right)$ & $1-a$ & $a$ & 1 \\
\hline Others $\left(\mathrm{n}_{1}\right)$ & $a$ & - & $a$ \\
\hline
\end{tabular}

The discussion carried out so far concludes as follows: In case of benchmarking the nuclear data with a measured neutron or gamma-ray spectrum, if any discrepancy appears, one wants to know which energy neutrons are concerned for this discrepancy. Or before benchmark experiments, one wants to know in which energetic neutrons the expeiments benchmark. According to the present thought experiments, the benchmark sensitivity for the experiment is "equally" due not only to neutrons (named (A)) making neutrons conveying contribution directly to the deterctor, but also due to neutrons (B) making neutrons (A). In this case, it can be recognized that neutrons (B) convey the same contribution indirectly through (A), which is called indirect contribution. Similarly neutrons (C) making (B) have the same indirect contribution as (B) and so on, i.e., all the ancestor neutrons have the same contribution (error sensitivity). This gives us an very important hint for the benchmark error sensitivity as in the following section:

\subsection{Benchmark sensitivity with Lewins importance in forward Monte Carlo calculations}

As Lewins proposed ${ }^{4)}$, in particle transport calculations, importance is regarded as contribution each particle has. The Lewins' contribution is defined as all the contribution which will be made by all the progenies of the particle in the whole transport calculation. According to the present thought experiments, it was found that the contribution governing the error sensitivity in Table 3 was exactly the same as the Lewins's contribution (importance). It means that benchmark error sensitivity could be evaluated by importance which can be estimated by forward Monte Carlo calculations. It also means error sensitivity could be predicted in forward Monte Carlo calculations, i.e., without adjoint function.

As a next phase, a more theoretical approach to form the mathematical expression with the present thought experiment and Lewins' style importance. And practical Monte Carlo calculations are planned to carry out to confirm the validity.

\section{Conclusion}

Thought experiments have been conducted to discuss how to estimate performance of benchmark experiments for neutron nuclear data for fusion reactor design. We carried out simple and precise thought experiments to examine which energy neutron is deeply concerned to the discrepancy, in case that there appears discrepancy between an experiment and its analysis with a transport code. As a result of the thought experiments, the benchmark sensitivity is "equally" due not only to neutrons (named (A)) making neutrons conveying contribution directly to the deterctor, but also due to neutrons (B) making neutrons (A). The neutrons (B) convey the same contribution indirectly through (A), which is called indirect contribution. Similarly all the ancestor neutrons have the same contribution (error sensitivity), which is an indirect contribution.

The above result leads the following conclusion: Because the Lewins's contribution (importance) is exactly the same as contribution governing the error sensitivity, benchmark error sensitivity could be evaluated by importance which can be estimated by forward Monte Carlo calculations. It also means error sensitivity could be predicted in forward Monte Carlo calculations, i.e., without adjoint function. With this procedure, it would become possible that from a sensitivity analysis in advance we could know how well and which energy nuclear data could be benchmarked with a benchmark experiment.

\section{References}

1) MCNP Team, "MCNP5-1.40 RSICC Release Notes", $L A$ UR-05-8617 (2005).

2) M. Ohta et al., "Preliminary spectrum shifter design for 
intermediate energy nuclear data benchmark experiments with DT neutrons," Fusion Eng. Des., 84, 1446-1449 (2009).

3) I. Murata, M. Ohta, H. Miyamaru, K. Kondo, S. Yoshida, T. Iida, K. Ochiai, C. Konno, "Performance Analysis of Fusion Nuclear-data Benchmark Experiments for Light to Heavy Materials in MeV Energy Region with A Neutron Spectrum Shifter," J. Nucl. Mater., 417, 1127-1130(2011).

4) J. Lewins, "IMPORTANCE The Adjoint Function", PERMAMON PRESS (1965). 